\title{
Simulation of the Microstructural Evolution of Pure Material and Alloys in an Undercooled Melts via Phase-field Method and Adaptive Computational Domain
}

\author{
Alexandre Furtado Ferreira ${ }^{a *}$, Ivaldo Leão Ferreira ${ }^{a}$, \\ Janaan Pereira da Cunha ${ }^{a}$, Ingrid Meirelles Salvino ${ }^{a}$ \\ ${ }^{a}$ Universidade Federal Fluminense - UFF, Volta Redonda, Rio de Janeiro, Brazil
}

Received: April 29, 2014; Revised: April 28, 2015

\begin{abstract}
The phase-field methods were developed mainly for studying solidification of pure materials, being then extended to the solidification of alloys. In spite of phase-field models being suitable for simulating solidification processes, they suffer from low computational efficiency. In this study, we present a numerical technique for the improvement of computational efficiency for computation of microstructural evolution for both pure metal and binary alloy during solidification process. The goal of this technique is for the computational domain to grow around the microstructure and fixed the grid spacing, while solidification advances into the liquid region. In the numerical simulations of pure metal, the phase-field model is based on the energy and phase equations, while, for binary alloy, the said model is based on the concentration and phase equations. Since the thermal diffusivity in the energy equation is much larger than the diffusivity term in phase equation in pure metal system, about twenty eight times the difference between them. The computational domain growth around the microstructure is controlled according with the thermal diffusivity for pure material in the liquid region. In the numerical simulation of dendritic evolution of $\mathbf{F e}-\mathbf{C}$ alloy, the idea is similar, i.e., the solute diffusivity in concentration equation is larger than the diffusivity term in phase equation in the liquid region, in this case eleven times the difference in $\mathbf{F e}-\mathbf{C}$ alloy system. The computational domain growth is controlled via solute diffusivity in the liquid region. Hence, phase-field model is proposed with an adaptive computational domain for efficient computational simulation of the dendritic growth in a system for both pure metal and binary alloy. The technique enables us to reduce by about an order of magnitude the run time for simulation of the solidification process. The results showed that the microstructure with well-developed secondary arms can be obtained with low computation time.
\end{abstract}

Keywords: computer simulation, dendrites, numerical efficiency

\section{Introduction}

The understanding and modeling of dendritic growth has remained a central theme of solidification research for many years. Understanding the solidification process is of great importance because the resulting microstructures determine the properties of the material. Although there have been significant developments in understanding dendritic structures in the past decades, our knowledge of the dendritic growth is based on experiments and idealized theoretical models. On the other hand, phase-field models are known to be very powerful in describing non-equilibrium dendritic evolution. They are very efficient because, in the numerical treatment based on them, all the governing equations are written for the whole domain without distinguishing the interface from the solid and liquid phases. Furthermore, direct tracking of the interface position is not needed during numerical simulation of the solidification process. The phase-field models were developed mainly for studying solidification of pure materials ${ }^{1}$, being then extended to the solidification of binary ${ }^{2}$, ternary ${ }^{3}$, and quaternary in Salvino et al. ${ }^{4}$ alloys.

*e-mail: furtado@metal.eeimvr.uff.br
Recently, Xu et al. ${ }^{5}$ used phase-field models focused on pure materials. Their paper presented a detailed numerical method and algorithm for solving two-dimensional (2-D) phase-field model. Comparison between the fully-coupled and sequential techniques showed that CPU time of the second approach is approximate $10 \%$ greater than that of the first one. However, the sequential method is chosen for computations in order to reduce storage requirements as much as possible. The authors found that the numerical results capture well the complex physics of the solidification problem. Consistent with physical reality, the computed critical radius indicates existence of a critical value for a nucleus to grow in the phase field simulation. Moreover, the critical radius decreases linearly with increasing Stefan number, which means that, if the Stefan number is large enough, solidification always takes place, no matter what the initial conditions are. In addition, they studied dendrite shapes at different degrees of supercooling; the results are in agreement with the experimental results.

Moelans et al. ${ }^{6}$ published a paper presenting an introduction to phase-field models and an overview of their possibilities. Amongst those, as listed by the authors, 
was the simulation of solidification processes, precipitate growth and, more recently, an application to solid-state phase transformations like the austenite-to-ferrite transformation in steels, dislocation dynamics, as well as crack nucleation and propagation. Achievements are expanding rapidly, due to improved modeling and implementation techniques and growing computer capacity.

From a somewhat more theoretical standpoint, it should be noted that the interface morphology of the solidification front calculated by phase-field models reproduces the known patterns of a dendrite structure. The state of the domain is represented by the distribution of a single variable known as the order parameter, $\phi$, or phase-field variable. In this study, the solid state is represented by $\phi=+1$, while, in the liquid region, $\phi=0$. The region in which $\phi$ changes progressively from +1 to 0 is defined as the solid/liquid interface. In spite of phase-field models being suitable for simulating solidification processes, as mentioned by Moelans et al. ${ }^{6}$, they suffer from low computational efficiency. For example, for computation of a dendrite with side-branches, the computational domain should be discretized into one million points. Thus, the computational stability condition in an explicit finite scheme can be guaranteed only with a very small time step. Xu et al. ${ }^{5}$ show a sequential method to reduce required storage during the calculations of the solidification process. In this study, on the other hand, we present a numerical technique for the improvement of computational efficiency for computation of dendritic evolution in solidification processes for both pure material (Ni) and binary alloy ( $\mathbf{F e}-\mathbf{C})$. In both cases presented in this article, at the start of the solidification process, there is a solid nucleus placed in the very small computational domain. The goal of this technique is for the computational domain to grow around the dendrite and fixed the grid spacing, while solidification advances into the liquid. The transient response of the phase equation is controlled by the product M. $\varepsilon^{2}$. This parameter acts in the phase-field model similarly to the thermal diffusivity (D) in the energy transport equation. Since the thermal diffusivity is much larger than the product M. $\varepsilon^{2}$, in pure metal system, for example twenty eight times difference in pure material system, the effect of heat transfer first occurs in the pure metal system. This way, the growth around the dendrite is controlled according with the thermal diffusivity (D) in liquid region. For numerical simulation of the dendritic evolution of binary alloy (Fe-C), the idea is similar to the pure metal $(\mathbf{N i})$. In other words, the solute diffusivity in liquid region $\left(\mathbf{D}_{\mathbf{L}}\right)$ is larger than said product $\mathbf{M}-\varepsilon(\theta)^{2}$, so the growth of the domain around the dendrite is controlled via solute diffusivity in liquid. The numerical technique for both cases pure metal (Ni) and alloy (Fe-C) enables us to reduce the run time in simulation of dendritic evolution during solidification process.

\section{Phase-field Modeling}

\subsection{Phase-field modeling for pure materials}

The phase-field model is based on the simultaneous solution of energy and phase equations for pure materials. Phase-field modeling assumes the growth of seeds in the liquid phase. According to this hypothesis, there are three regions to be considered: the solid nucleus, the liquid phase and the solid/liquid interface. The state of the entire domain is represented by the distribution of a single variable known as the order parameter, $\phi$, or phase-field variable. The region in which $\phi$ changes from 1 to 0 is defined as the solid/liquid interface. The time evolution equation of the phase-field $\phi$ is described by ${ }^{1}$ :

$$
\begin{aligned}
& \frac{1}{\mathrm{M}} \cdot \frac{\partial \phi}{\partial \mathrm{t}}=\left(\nabla \cdot\left(\varepsilon(\theta)^{2} \nabla \phi\right)+\right. \\
& \frac{\partial}{\partial \mathrm{y}}\left(\varepsilon(\theta) \varepsilon^{\prime}(\theta) \frac{\partial \phi}{\partial \mathrm{x}}\right)-\frac{\partial}{\partial \mathrm{x}}\left(\varepsilon(\theta) \varepsilon^{\prime}(\theta) \frac{\partial \phi}{\partial \mathrm{y}}\right)- \\
& \mathrm{wg}^{\prime}(\phi)-\mathrm{h}^{\prime}(\phi) \frac{\Delta \mathrm{H}}{\mathrm{T}_{\mathrm{m}}}\left(\mathrm{T}-\mathrm{T}_{\mathrm{m}}\right)
\end{aligned}
$$

where $\mathbf{M}$ is defined as the solid/liquid interface mobility, the angle $\theta$ is given by the orientation of a vector perpendicular to the solid/liquid interface, e.g., $\nabla \phi . \Delta \mathrm{H}$ is the latent heat and $\mathbf{T}_{\mathrm{m}}$ the melting temperature. The function $\mathbf{g}^{\prime}(\phi)$ that multiplies $\mathbf{w}$ determines the distribution of the excess free-energy at the interface. $\mathbf{h}(\phi)$ is a function that satisfies the condition $h^{\prime}(0)=h^{\prime}(1)=0$. As in reference Moelans et al. ${ }^{6}$, we chose

$$
\begin{aligned}
& \mathrm{h}(\phi)=\phi^{3}\left(10-15 \phi+6 \phi^{2}\right) \\
& \mathrm{g}(\phi)=\phi^{2}(1-\phi)^{2}(3)
\end{aligned}
$$

The method most widely used to include anisotropy for a two-dimensional calculation is to assume that $\varepsilon$ in Equation 1 depends on $\theta$, the orientation of the normal to the interface with respect to the $\mathrm{x}$-axis:

$$
\varepsilon(\theta)=\varepsilon\left(1+\delta_{\varepsilon} \operatorname{cosj}\left(\theta-\theta_{0}\right)\right)
$$

where $\delta_{\varepsilon}$ is the anisotropy constant. The value of $\mathbf{j}$ controls the number of preferential directions of the material's anisotropy, equaling 0 for the isotropic cases, 4 for anisotropy of 4 directions, and so on. The constant $\theta_{\mathbf{o}}$ is the interface orientation with respect to the maximum anisotropy, while $\varepsilon$ and $\mathbf{w}$ are parameters associated with the interfacial energy $(\sigma)$ and interface thickness $(\lambda)$, as proposed by Boettinger et al. ${ }^{7}$ :

$$
\sigma=\frac{\varepsilon \sqrt{w}}{3 \sqrt{2}}
$$

$2 \lambda \cong \frac{2.2 \sqrt{2 \varepsilon}}{\sqrt{w}}$

For the interface mobility, we follow references Ferreira et al. ${ }^{1}$ and Boettinger et al. ${ }^{7}$ :

$$
\mathrm{M}=\frac{\mathrm{T}_{\mathrm{m}} \mu}{2.73 \Delta \mathrm{H} \lambda}
$$

where $\mu$ is the linear interface kinetic coefficient. The phase-field model particularized for a pure material, subjected to a nonuniform thermal field, includes an energy transport equation:

$$
\frac{\partial \mathrm{T}}{\partial \mathrm{t}}=\mathrm{D} \nabla^{2} \mathrm{~T}+\frac{\Delta \mathrm{H}}{\rho \mathrm{C}_{\mathrm{p}}} \mathrm{h}^{\prime}(\phi)
$$

In Equation 8, D is the thermal diffusivity, as previously indicated in Equation $1 \Delta \mathrm{H}$ is the latent heat, considered 
positive for solidification, $\rho$ is the material's density, assumed to be the same both solid and liquid phase, and $\mathbf{C}_{\mathbf{p}}$ is the specific heat.

\subsection{Phase-field modeling for binary alloy}

The phase-field equation for simulates the solidification process for binary alloy is described by ${ }^{2}$ :

$$
\begin{aligned}
& \frac{1}{\mathrm{M}} \cdot \frac{\partial \phi}{\partial \mathrm{t}}=\nabla \cdot\left(\varepsilon(\theta)^{2} \nabla \phi\right)+ \\
& \frac{\partial}{\partial \mathrm{y}}\left(\varepsilon(\theta) \varepsilon^{\prime}(\theta) \frac{\partial \phi}{\partial \mathrm{x}}\right)-\frac{\partial}{\partial \mathrm{x}}\left(\varepsilon(\theta) \varepsilon^{\prime}(\theta) \frac{\partial \phi}{\partial \mathrm{y}}\right)- \\
& \mathrm{wg}^{\prime}(\phi)+\frac{\mathrm{RT}}{\mathrm{V}_{\mathrm{m}}} \mathrm{h}^{\prime}(\phi) \operatorname{Ln}\left(\frac{1-\mathrm{C}_{\mathrm{S}}^{\mathrm{eq}}}{1-\mathrm{C}_{\mathrm{L}}^{\mathrm{eq}}} \cdot \frac{1-\mathrm{C}_{\mathrm{L}}}{1-\mathrm{C}_{\mathrm{S}}}\right)
\end{aligned}
$$

The evolution of the solid nucleus with time $(\partial \phi / \partial t)$ is assumed to be proportional to the variation of the free-energy functional with respect to the order parameter, $\phi$. The terms of the phase equation are derived from this free-energy functional, which must decrease during any solidification process, as indicated in the article by Salvino et al. ${ }^{4}$. The last product on the right-hand side translates the driving force behind the solidification process. Here, $\mathbf{R}$ is the gas constant and $\mathbf{V}_{\mathbf{m}}$, the molar volume. The arguments to the natural logarithms, and, are, respectively, the equilibrium concentrations of carbon in the solid and liquid region. Their respective ordinary concentrations in the liquid and solid regions are denoted, by the pairs $\mathbf{C}_{\mathbf{L}}$ and $\mathbf{C}_{\mathbf{S}}$.

As proposed by Ode et al. ${ }^{2}$, concentrations of carbon in both regions is calculated with the solute transport equation, numbered (10),

$$
\begin{aligned}
& \frac{\partial \mathrm{c}}{\partial \mathrm{t}}=\nabla\left(\mathrm { D } ( \phi ) \left\{(1-\mathrm{h}(\phi)) \cdot \mathrm{c}_{\mathrm{L}}\left(1-\mathrm{c}_{\mathrm{L}}\right)+\right.\right. \\
& \left.\left.\mathrm{h}(\phi) \mathrm{c}_{\mathrm{S}}\left(1-\mathrm{c}_{\mathrm{S}}\right)\right\} \operatorname{Ln}\left(\frac{\mathrm{c}_{\mathrm{L}}}{1-\mathrm{c}_{\mathrm{L}}}\right)\right)
\end{aligned}
$$

In this equation, $\mathbf{D}(\phi)$ is the carbon diffusivity in the solid and liquid regions. The model used here takes into account solute diffusivity in the liquid and interface regions.

The model parameters $\varepsilon$ and $\mathbf{w}$ for binary alloys are calculated in the same way as proposed by Boettinger et al. ${ }^{7}$, Equations 5 and 6. From Salvino et al. ${ }^{4}$, the phase-equation mobility for binary alloys, $\mathbf{M}$, is computed as

$$
\frac{1}{\mathrm{M}}=\frac{\varepsilon^{3}}{\sigma \sqrt{2 \mathrm{w}}} \cdot\left(\frac{1}{\mathrm{D}_{\mathrm{L}}} \cdot \xi\left(\mathrm{C}_{\mathrm{L}}^{\mathrm{Eq}}, \mathrm{C}_{\mathrm{S}}^{\mathrm{Eq}}\right)\right.
$$

where the $\xi\left(\mathbf{C}_{\mathbf{L}}^{\mathbf{E q}}, \mathbf{C}_{\mathbf{S L}}^{\mathbf{E q}}\right)$ is obtained from

$$
\begin{aligned}
& \xi\left(\mathrm{C}_{\mathrm{L}}^{\mathrm{Eq}}, \mathrm{C}_{\mathrm{S}}^{\mathrm{Eq}}\right)=\frac{\mathrm{RT}}{\mathrm{V}_{\mathrm{m}}}\left(\mathrm{C}_{\mathrm{L}}^{\mathrm{Eq}}-\mathrm{C}_{\mathrm{S}}^{\mathrm{Eq}}\right)^{2} \times \\
& \int_{0}^{1} \frac{\mathrm{h}(\phi)[1-\mathrm{h}(\phi)]}{[1-\mathrm{h}(\phi)] \mathrm{C}_{\mathrm{L}}^{\mathrm{Eq}}\left(1-\mathrm{C}_{\mathrm{L}}^{\mathrm{Eq}}\right)+\mathrm{h}(\phi) \mathrm{C}_{\mathrm{S}}^{\mathrm{Eq}}\left(1-\mathrm{C}_{\mathrm{S}}^{\mathrm{Eq}}\right)} \cdot \frac{\mathrm{d}(\phi)}{\phi(1-\phi)}
\end{aligned}
$$

In Equations 11 and 12, $\mathbf{L}$ and $\mathbf{S}$ stand for liquid and solid, respectively.

\section{Numerical Simulation}

To simulate growth of an asymmetrical dendrite of pure materials and binary alloys, it is necessary to introduce a noise term in the right-hand side of the phase-field equations. A usual expression for this noise, as indicated by Ferreira et al. ${ }^{1}$, is

$$
\text { noise }=16 \operatorname{ar} \phi^{2}(1-\phi)^{2}
$$

where $\mathbf{r}$ is a random number between -1 and +1 . The "a" parameter is the noise amplitude. Maximum noise corresponds to $\phi=0.5$, at the center of the interface, whereas at $\phi=0$ (liquid region) and $\phi=+1$ (solid region) there occurs no noise. That is to say, noise is generated at the interface.

Equations 1, 8, 9 and 10 were solved by an explicit finite-difference method, with a mesh sufficiently refined to describe details of the dendrites. Performing the computations with a numerical grid of $200 \times 200$ points with parameters determined in the previous section and the physical properties of nickel, it was not feasible to obtain a dendrite with developed secondary arms, due to the small computation domain. Dendrites with fully developed side branches necessitate a computational domain with several million points. However, computation with such a large computational domain is restricted not only by the computational efficiency, but also by memory size. In the present study, we develop a numerical technique in order to improve efficiency. The idea was originally proposed for simulating the dendrite growth from an undercooled pure melt and has been extended to solidification of binary alloy. In pure metal case (Ni), the thermal diffusivity, in Equation 8 , is much larger than the product $\mathbf{M}-\varepsilon(\theta)^{2}$, in Equation 1 , for example about twenty eight times difference in pure material system. Therefore, first to occur is the effect of the heat transfer, then phase change during simulation of solidification process. A greater value of $\mathbf{D}$ (Equation 8, for pure materials) forces the thermal front to be always ahead of the solidification interface. Hence, there is always a thermal gradient ahead of the solidification front. In this study for solidification of pure materials, the thermal boundary layer is defined as a region with

$$
\mathrm{T}(\mathrm{I}, \mathrm{J})>\mathrm{T}_{0}+1.1
$$

where $\mathbf{T}_{0}$ is a given initial undercooled temperature. If the condition $\mathbf{T}(\mathbf{I}, \mathbf{J})>\mathbf{T 0}+\mathbf{1 . 1}$ at $\mathbf{( I , J )}$ in the square region is satisfied, the adaptive computational domain grows around the dendrite. Whenever the condition is satisfied, new temperature $\mathbf{T}(\mathbf{I}, \mathbf{J})$ and phase $\boldsymbol{\phi}(\mathbf{I}, \mathbf{J})$ at the current time step are calculated from the explicit finite-difference method from the values in previous steps, while the computational domain grows one unit of points on the $x-y$ directions. If the condition just stated is not satisfied, the computational domain does not grow. The new temperature $\mathbf{T}(\mathbf{I}, \mathbf{J})$ and phase $\phi(\mathbf{I}, \mathbf{J})$ are then calculated from the values in previous steps for a small-size domain. In the simulation of binary alloy (Fe-C) solidification, the idea is similar to the pure metal (Ni). In binary alloy case, the phase-field model is based on the phase and concentration equations. The phase equation includes the product $\mathbf{M}-\boldsymbol{\varepsilon}(\boldsymbol{\theta})^{2}$, and $\mathbf{D}(\boldsymbol{\phi})$ is the carbon diffusivity in the concentration equation. The 
solute diffusivity in liquid phase $\left(\mathbf{D}_{\mathbf{L}}\right)$ (Equation 10) is larger than the product $\mathbf{M}-\boldsymbol{\varepsilon}(\boldsymbol{\theta})^{2}$ (Equation 9), about eleven times is the difference between them. Hence, first to occur is the concentration change, then the phase change during simulation of solidification in binary alloy system. In other words, during solidification, the solutes are rejected into the liquid phase, which then becomes rich in solute just ahead of the interface. The ahead of the interface there is a thickness of the diffusion boundary layer in the liquid due the high mobility of solute in said region. As mobility solute is greater as compared to that front solidification, it forces the gradient concentration in liquid to be always ahead of the solidification interface. In the solidification of binary alloys, the diffusion boundary layer is defined as

$\mathrm{C}(\mathrm{I}, \mathrm{J})>\mathrm{C}_{0}+1 \times 10^{-3}$

where $\mathbf{C}_{\mathbf{0}}$ is a given initial concentration. If the condition is satisfied $\mathbf{C}(\mathbf{I}, \mathbf{J})>\mathbf{C}_{\mathbf{0}}+\mathbf{1} \times \mathbf{1 0}^{-\mathbf{3}}$, the adaptive domain grows around the binary alloy dendrite; otherwise, it does not grow. The block diagram in Figure 1 shows the flow of information in the numerical program for both pure materials (Figure 1a) and binary alloy (Figure 1b).

The calculations were performed on an Intel ${ }^{\circledR} 2$ Quad processor, with 1.38GB RAM. In this study, in the initial stage of solidification the computational domain used to calculate dendrite evolution is rather small, about $200 \times 200$ points. Both phase-field models presented in this article, one with an adaptive computation domain and one with a computation domain of fixed size, were constructed with the same grid spacing $\left(\mathbf{d x}=\mathbf{d y}=\mathbf{2} \times \mathbf{1 0}^{-\mathbf{8}} \mathbf{m}\right)$. The difference is in the number of nodes of the computational domain. In the first model, the computational domain is very small in the initial steps of the computation; consequently the computational efficiency is improved and the memory size requirement is reduced. In the second model with a computation domain of fixed size, in the initial steps of the computation the computational domain

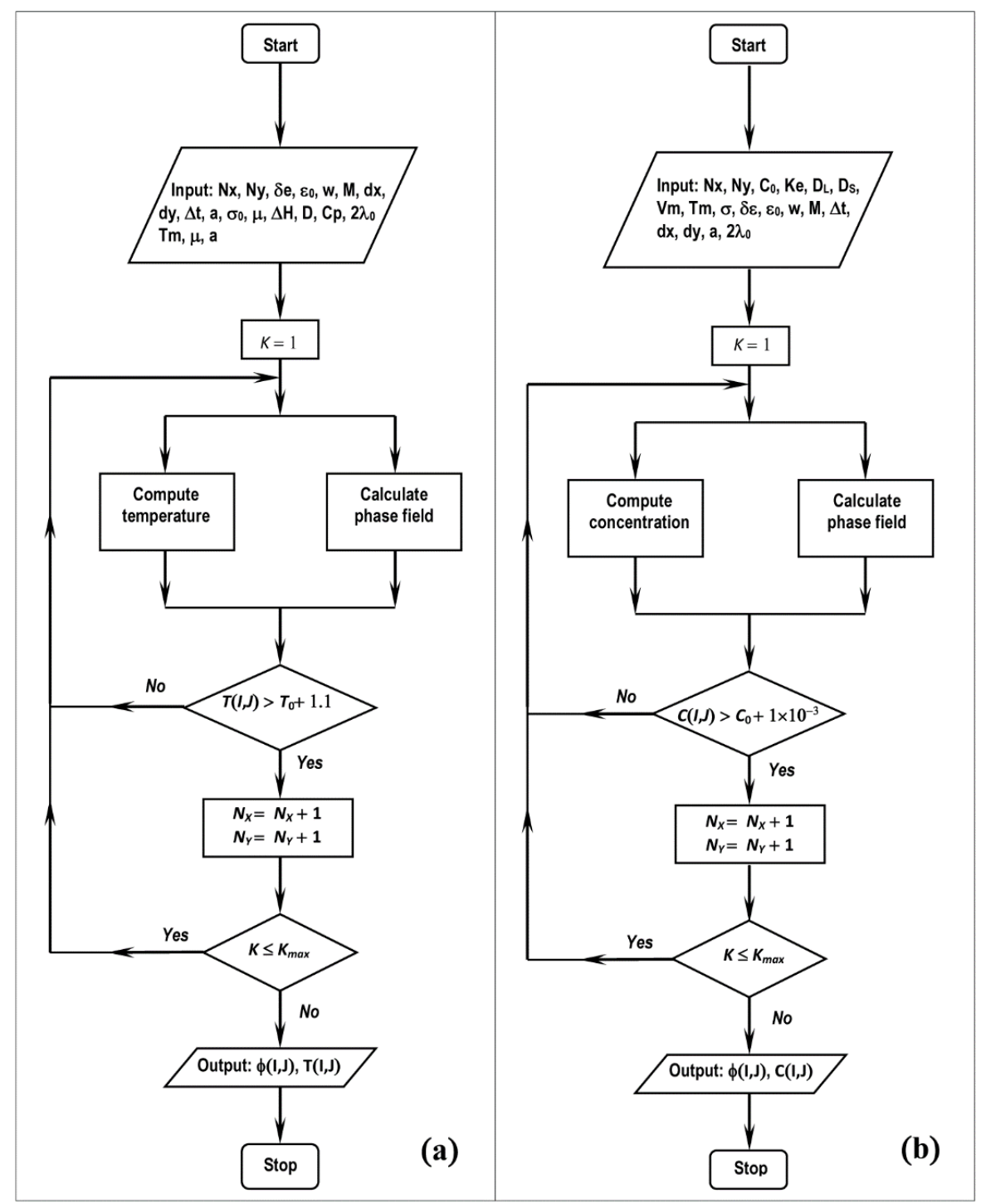

Figure 1. The phase-field model with adaptive computational domain. (a) pure metal and (b) binary alloy. 
is very large, thereby reducing computational efficiency and the memory size requirement is increased.

The phase, energy and concentration equations were solved in a computational domain divided into square grids of $\mathbf{I} \times \mathbf{J}$, with a grid spacing of $\mathbf{d x}=\mathbf{d y}$ not only for the thermal field, but also for calculation of the phase field and concentration. The thermal field T(I,J ), phase-field $\phi(\mathbf{I}, \mathbf{J})$ and concentration $\mathbf{C}(\mathbf{I}, \mathbf{J})$ at each point in the grid $(\mathbf{I}, \mathbf{J})$ are known from the previous step, by the explicit finite-difference. For each point $\mathbf{I}, \mathbf{J}$ in the grid, a real value $\phi(\mathbf{I}, \mathbf{J})$, describes the phase state of the grid, is assigned $\phi(\mathbf{I}, \mathbf{J})=\mathbf{0}$ to indicate the grid in the liquid state and $\phi(\mathbf{I}, \mathbf{J})=+\mathbf{1}$ for the solid state. The ensemble of all grid points with $0.001<\phi(\mathbf{I}, \mathbf{J})<\mathbf{0 . 9 9 9}$ is taken to represents the interface region.

\section{Results and Discussion}

In order to test the computational efficiency of our numerical technique, we compared the computer run time for the calculation of dendritic growth in undercooled melts using a phase-field model with and without an adaptive computational domain. We analyzed the computational efficiency for both cases pure material (Ni) and binary alloy (Fe-C).

\subsection{Phase-field simulation of dendritic solidification for pure material}

The parameters and properties adopted in this study for pure materials (Ni) are summarized in Tables 1 and 2, respectively. The phase-field mobility $(\mathbf{M})$ for pure metal is calculated by Equation 7.

To exhibit the similarities between the dendrites of nickel calculated in the present paper and those described in the literature, we introduce Figure 2. In that figure, both pictures display: a) the secondary arms; b) the secondary arm increase with distance behind the primary dendrite tip; c) the asymmetry of the side branch found in the secondary arms; and d) the secondary arms growth rigorously perpendicular to the primary arm.

Figure 3 shows temperature profiles and the phase-field variable across solid, interface and liquid regions. In this simulation, the solid/liquid interface advances into the liquid region. The transient response of phase-field equations is controlled by the product $\mathbf{M - \varepsilon}(\boldsymbol{\theta})^{2}$, in Equation 1. This parameters act in the phase-field model similarly to the thermal diffusivity $\mathbf{D}$ in the thermal energy Equation 8. As Kim et al. ${ }^{8}$ pointed out, in the formation of a dendritic morphology in pure metals, it is important that the thermal diffusivity becomes greater than its similar term, $\mathbf{M - \varepsilon}(\boldsymbol{\theta})^{2}$. This can be explained by analyzing Figure 3 . The greater value of $\mathbf{D}$ forced the thermal front to be always ahead of the solidification interface. Hence, there was always a thermal gradient ahead of interface. In pure metal case (Ni), the thermal diffusivity (D), in Equation 8, is much larger than the product $\mathbf{M}-\boldsymbol{\varepsilon}(\boldsymbol{\theta})^{2}$, in Equation 1, for example about twenty eight times difference in pure material system. On the other words, first of all occur the temperature change, and then the liquid becomes solid region. Hence, the present paper is based in that approach, i.e., if the temperature change occurs, the adaptive computational domain grows
Table 1. Model parameters (Ni).

\begin{tabular}{lc}
\hline Anisotropy constant, $\delta_{\varepsilon}$ & 0.025 \\
Coefficient of phase-field gradient & $2.01 \times 10^{-4}(\mathrm{~J} / \mathrm{m})^{1 / 2}$ \\
energy term, $\varepsilon_{\mathrm{o}}$ & \\
Free energy factor, $w$ & $0.61 \times 10^{8} \mathrm{~J} / \mathrm{m}^{3}$ \\
Phase-field mobility, $M$ & $13.47 \mathrm{~m}^{3} / \mathrm{sec} \cdot \mathrm{J}$ \\
Grid spacing, dx & $2 \times 10^{-8} \mathrm{~m}$ \\
Grid spacing, dy & $2 \times 10^{-8} \mathrm{~m}$ \\
Time step, $\Delta t$ & $1 \times 10^{-12} \mathrm{sec}$ \\
Noise amplitude factor, $a$ & 0.025 \\
\hline
\end{tabular}

Table 2. Material properties of $\mathrm{Ni}^{8}$.

\begin{tabular}{lc}
\hline Interface energy, $\sigma_{\mathrm{o}}$ & $0.37 \mathrm{~J} / \mathrm{m}^{2}$ \\
Kinetic coefficient at interface, $\mu$ & $2 \mathrm{~m} / \mathrm{s} . \mathrm{K}$ \\
Melting temperature, $T_{M}$ & $1728 \mathrm{~K}$ \\
Latent heat, $\Delta H$ & $2.35 \times 10^{9} \mathrm{~J} / \mathrm{m}^{3}$ \\
Thermal diffusivity, $D$ & $1.55 \times 10^{-5} \mathrm{~m}^{2} / \mathrm{s}$ \\
Specific heat, $C_{P}$ & $5.42 \times 10^{6} \mathrm{~J} / \mathrm{m}^{3} . \mathrm{K}$ \\
Interface width, $2 \lambda_{\mathrm{o}}$ & $8 \times 10^{-8} \mathrm{~m}$ \\
\hline
\end{tabular}
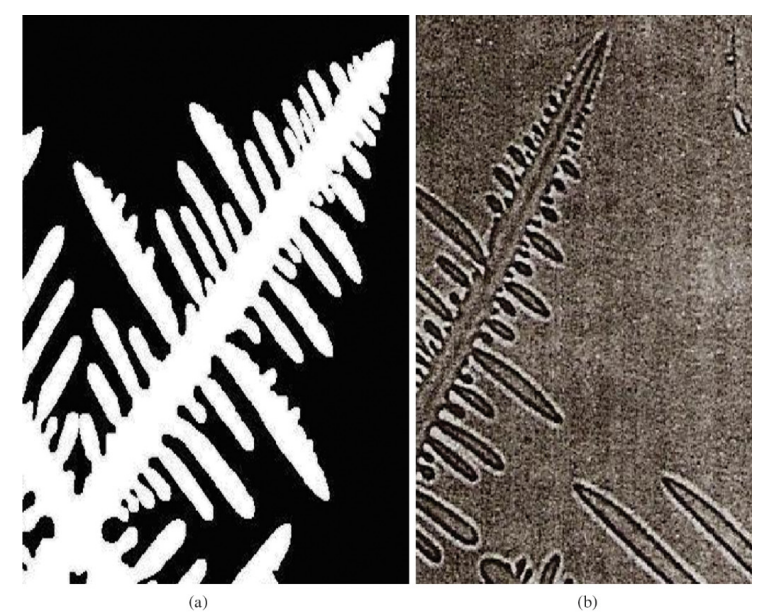

Figure 2. (a) Present calculation and (b) dendrite found in the literature, Prates ${ }^{11}$.



Figure 3. Temperature and phase-field variable profiles across solid, interface and liquid regions. 
around the dendrite; otherwise, the computational domain size is kept constant.

In order to test the computational efficiency of our numerical technique, we compared the computer run time for calculation of dendritic growth in undercooled melts using a phase-field model with and without an adaptive computational domain. Figure 4 shows the dendrite growth obtained by the phase-field model with the adaptive computational domain for different solidification times and domain sizes.

Figures $4 \mathrm{a}-\mathrm{d}$ shows the development of the adaptive computational domain for dendritic growth. In Figure 4a, the dendrite started to grow from a nucleus added at the center of the computational domain with $85 \times 85$ points, solidification time being equal to $4.47 \times 10^{-9} \mathrm{sec}$, insufficient for growth of primary and secondary arms. In Figure $4 b$, the numerical grid $(205 \times 205)$ is larger than that of Figure $4 \mathrm{a}$ $(85 \times 85)$, due to the dendrite tip advancing into supercooled liquid during the solidification process. In Figure $4 b$, one observes only primary arms, with no side branching, for a solidification time of $3.62 \times 10^{-8} \mathrm{sec}$. In Figure $4 \mathrm{c}$, the time for solidification $\left(9.35 \times 10^{-8} \mathrm{sec}\right)$ is sufficient for the growth of secondary arms. Finally, in Figure 4d, the time for solidification is $1.50 \times 10^{-7} \mathrm{sec}$ and the numerical grid comprises $605 \times 605$ points. Here, it is possible to observe well-developed secondary arms away from the dendrite tip, while small side branches compete with each other shortly behind the dendrite tip. The asymmetry in the side branches is evinced in Figures 4c, d. This follows from the thermal field distribution. Again, side branching prefers the direction of latent heat release. In all of Figure 4, computational convergence is optimized through adoption of a small computational domain around the dendrite.

Figure 5 shows variation of computer run time (in seconds) as a function of primary dendrite length (in units of domain size). There, the open and solid circles are for the adaptive computational domain and a computational domain of fixed size, respectively. One can see that, with the adaptive computational domain, the run time required to reach a given primary dendrite growth is about a tenth of that with the computational domain of fixed size. Computational efficiency is guaranteed by using an adaptive computational domain for phase field and thermal calculation, in pure metal system. Because the computational domain is small at the beginning of the calculations, convergence is optimized.

Using the phase-field model with an adaptive computational domain for simulation of the solidification process, the calculation of dendritic growth is carried through with a computational domain sufficiently small for the phase field and thermal calculations. Increasing the primary dendrite length, one finds that the run-time versus primary-dendrite-length plot will tend to exhibit an exponential-like behavior.

\subsection{Phase-field simulation of dendritic solidification for binary alloy}

Table 3 presents the physical properties of the binary alloy used in the computations that follow. The parameters used in the phase-field model obtained of physical properties of the material were derived from Equations 5, 6, and 11 .

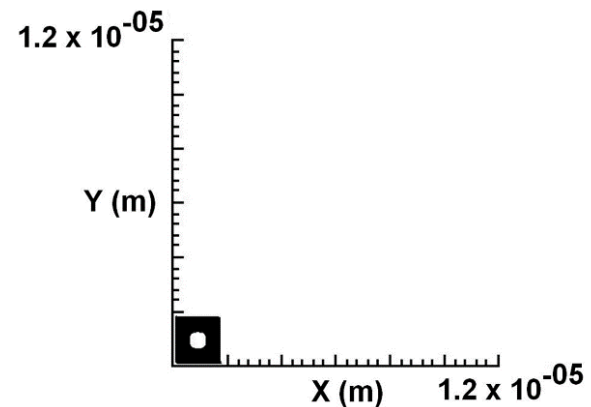

(a)



(c)

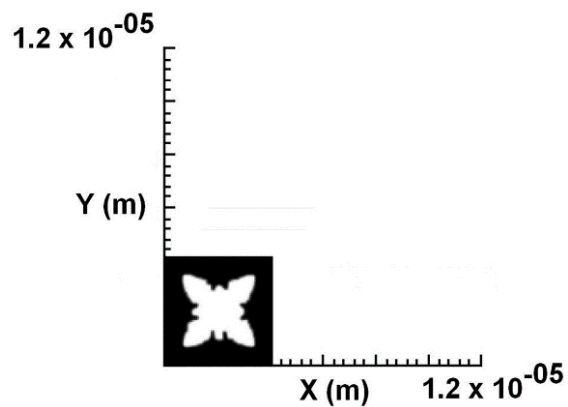

(b)

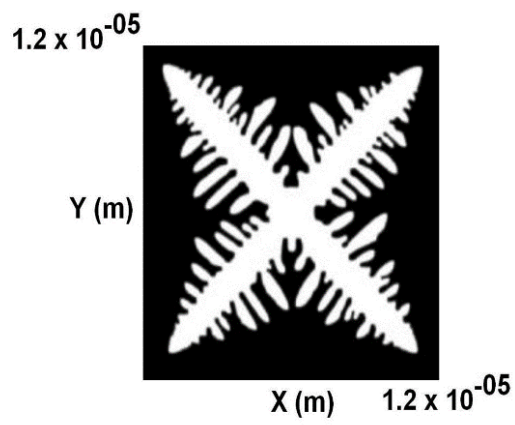

(d)

Figure 4. Development of the adaptive computational domain for dendritic growth. The numerical grids and solidification times are: (a) $85 \times 85$ points, $4.47 \times 10^{-9} \mathrm{sec}$; (b) $205 \times 205$ points, $3.62 \times 10^{-8} \mathrm{sec}$; (c) $405 \times 405$ points, $9.35 \times 10^{-8} \mathrm{sec}$; and (d) $605 \times 605$ points, $1.50 \times 10^{-7} \mathrm{sec}$. 
Table 4 presents these parameters. The phase-field mobility (M) for binary alloy (Fe-C) é calculated by Equations 11 and 12 . The boundary condition adopted for the phasefield model $(\phi)$ in this work is a zero-flux condition.

The Figure 6 shows the dendritic morphology obtained by both the phase-field model with adaptive computational domain (Figure 6a) and fixed domain (Figure 6b); one can see excellent agreement between the two cases. Figure 6 depicts the simulation of a Fe-C alloy calculated with a regular grid. In this simulation, a dendrite is presented with secondary arms. The secondary arms increase with the distance behind the primary dendrite tip. This was observed in experiments on dendritic growth in undercooled melts. The asymmetry in the side branches of the primary arms

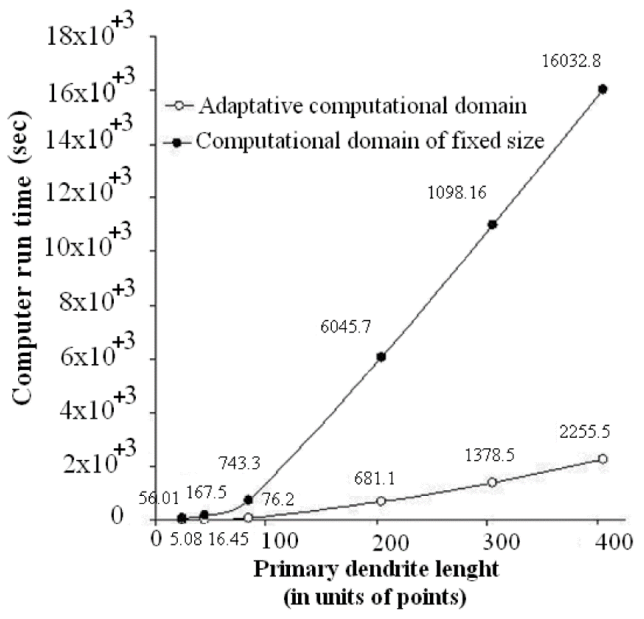

Figure 5. Variation of computer run time as a function of primary dendrite length, in units of points.

Table 3. Physical properties of the binary alloy analyzed. ${ }^{4}$

\begin{tabular}{lcc}
\hline \multicolumn{1}{c}{ Property } & C & Fe \\
\hline Initial concentration, $C_{0}$ & $6.93 \times 10^{-3} \mathrm{~mol} \%$ & - \\
Partition coefficient, $K_{E}$ & 0.17171 & - \\
Slope of liquids line, $M_{E}$ & $1772.77 \mathrm{~K} / \mathrm{mol}$ & - \\
Diffusivity in liquid phase, $D_{L}$ & $2.0 \times 10^{-.8} \mathrm{~m}^{2} / \mathrm{s}$ & - \\
Diffusivity in solid phase, $D_{S}$ & $6.0 \times 10^{-.9} \mathrm{~m}^{2} / \mathrm{s}$ & - \\
Molar volume, $V_{M}$ & - & $7.7 \times 10^{-6} \mathrm{~m}^{3} / \mathrm{mol}$ \\
Melting temperature, $T_{M}$ & - & $1811 \mathrm{~K}$ \\
Interface energy, $\sigma$ & - & $0.204 \mathrm{~J} / \mathrm{m}^{2}$ \\
\hline
\end{tabular}

Table 4. Computational parameters.

\begin{tabular}{lc}
\hline Anisotropy constant, $\delta_{\varepsilon}$ & 0.05 \\
Coefficient of phase-field gradient energy & $1.05 \times 10^{-.4}$ \\
term, $\varepsilon_{0}$ & $(\mathrm{~J} / \mathrm{m})^{1 / 2}$ \\
Free energy factor, $w$ & $6.73 \times 10^{7} \mathrm{~J} / \mathrm{m}^{3}$ \\
Phase-field mobility, $M$ & $0.166 \mathrm{~m}^{3} / \mathrm{s} . \mathrm{J}$ \\
Time step, $\Delta t$ & $1.0 \times 10^{-.8} \mathrm{~s}$ \\
Grid spacing, d $x$ & $2.0 \times 10^{-.8} \mathrm{~m}$ \\
Grid spacing, dy & $2.0 \times 10^{-.8} \mathrm{~m}$ \\
Noise amplitude factor, $a$ & 0.025 \\
\hline
\end{tabular}

observed in both Figure 6a-b is due to a noise source added to the phase-field equation.

In order to show the applicability of the phase-field model with adaptive computational domain, the influence of dendrite tip radius on the growth velocity is showed. The relationships between growth velocity and dendrite tip radius for a $\mathbf{F e}-\mathbf{C}$ alloy are shown in Figure 7. Data calculated by an analytical model, proposed by Stenanescu ${ }^{9}$, were plotted alongside for comparison. We can see that phase-field-based results lie above those obtained with analytical model proposed by Stefanescu. ${ }^{9}$ This may happen due to the evolution of the solid phase with time (Equation 9) is assumed to be dependent of the source term. This, in turn, depends of both concentrations in the solid and liquid region and temperature. The Stefanescu's model, on the other hand, takes into account tip radius and concentrations in the liquid region. One can see in Figure 7 that increasing the radius of dendrite tip influences the reduction of the dendritic growth velocity. The numerical results for the tip velocity are consistent with experimental conclusion, Altundas and Caginalp ${ }^{10}$, and compatible with the literature ${ }^{9}$, ${ }^{11}$ that tip velocity will decrease for larger radii.

Figure 8 exhibits the results of the carbon concentrations; we compare the results of a one-dimensional phase-field calculation with the predictions by Scheil's equation and by the Clyne-Kurz equation. The initial temperature of the computational domain is $1780 \mathrm{~K}$. For the calculations of carbon concentration, we assumed an isothermal
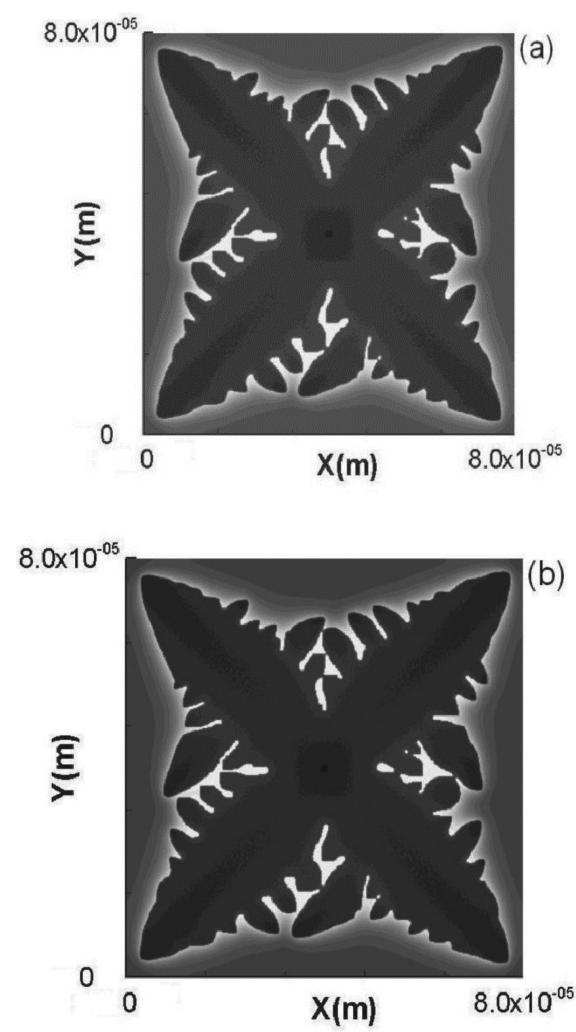

Figure 6. Comparison of dendritic morphology between phase-field model with adaptive computational domain (a) and fixed domain (b). 
solidification process. In the calculations, no anisotropy was imposed. The calculated carbon profile agrees better with the Clyne-Kurz equation than with Scheil's equation, when the solid fraction is above 0.5 , as shown in Figures 8 . This is because the Clyne-Kurz model assumed back-diffusion. In contrast, Scheil's analytical model neglected diffusion in the solid phase altogether, but assumed complete mixing of the solute in the liquid phase. $\mathbf{C}_{\mathrm{S}}$ is the concentration in the solid at the solid/liquid interface and $\mathbf{C}_{\mathbf{0}}$ is the initial concentration of solute. We can see that phase-field-based results lie above those obtained with Scheil's equation; the little difference in the early stage of solidification is due to the effect of the initial transient because of small diffusivity in the solid. These results show that the phase-field model is capable of computing the same solid concentration in the solid/liquid interface as estimated by Clyne-Kurz's equation. On the other hand, neither Scheil's nor the ClyneKurz equation is able to predict the composition profile in the solid. The phase-field model can simulate not only concentration in the solid but also a concentration profile in the liquid during solidification.

Plots in Figure 9 correspond to $4 \times 10^{-7} \mathrm{sec}$ of solidification time. The right-hand vertical axis gives the carbon concentration; the left-hand one, that of phase-field variable. When $\phi=+1$, we are in the solid region, whereas $\phi=0$ is the liquid. The interface lies between $\phi=+1$ and 0 . Therefore, one can see that the solid region is poor in carbon. This is because, during solidification, the solutes are rejected into the liquid phase, which then becomes rich in solute just ahead of the interface. As we move farther to the right, hence away from the interface, concentration decreases exponentially, towards their initial values in the liquid. Such tendency seems to be in agreement with the consideration that the Gibbs free energy is more negative in the solid phase. Still with respect to Figure 9, one can observe the carbon diffuse layer to be larger than that of phase, due to the greater diffusivity of concentration equation compared to that of phase. Whenever the changes for carbon concentration happen in liquid phase, in front of solid/liquid interface, the adaptive computational domain will grows around the dendrite.

In method similar done for the pure material, we test the computational efficiency of our numerical technique for binary alloy (Fe-C). So, were compared the computer run time for calculation of dendritic growth using a phase-field model with and without an adaptive computational domain.

Figures 10a-d shows the dendrite growth during the solidification process for binary alloy for different times and domain sizes.

In Figure 10a, a dendrite without secondary arms grows from center of the small computational domain with 85 points; solidification time is $1.33 \times 10^{-5} \mathrm{sec}$. In Figure $10 \mathrm{~b}$, the numerical grid with 205 points is larger than that of Figure 10a, due to the dendrite advancing into supercooled liquid. In Figure 10b, one observes only primary arms, with no side branching, the solidification time is $7.01 \times 10^{-5} \mathrm{sec}$. In Figure 10c, the time for solidification is $2.06 \times 10^{-4} \mathrm{sec}$ insufficient for we observe secondary arms well developed. Finally, in Figure 10d, the time for solidification is $1.50 \times 10^{-3} \mathrm{sec}$ and the numerical grid is 605 points. Here, it is possible to observe few developed secondary arms away from the dendrite tip during the solidification process. The asymmetry in the side branches is evinced in Figures 10d. In all of Figure 10, computational convergence is optimized through adoption of a small computational domain around the dendrite.

Figure 11 shows variation of computer run time (in seconds) as a function of primary dendrite length (in

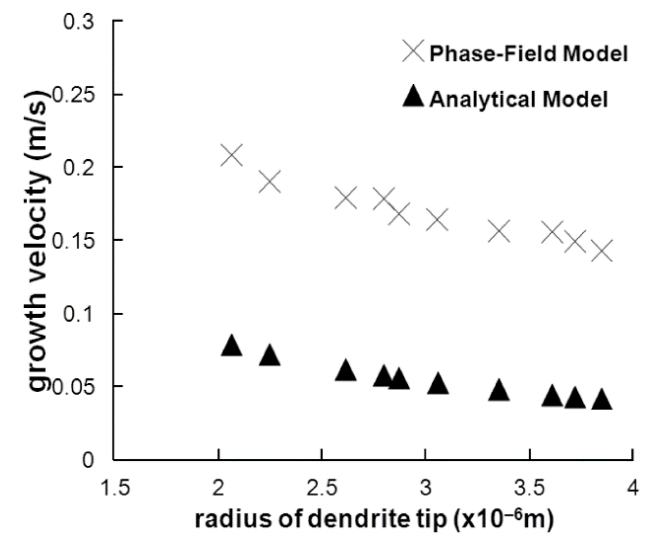

Figure 7. Growth velocity versus radius of dendrite tip.

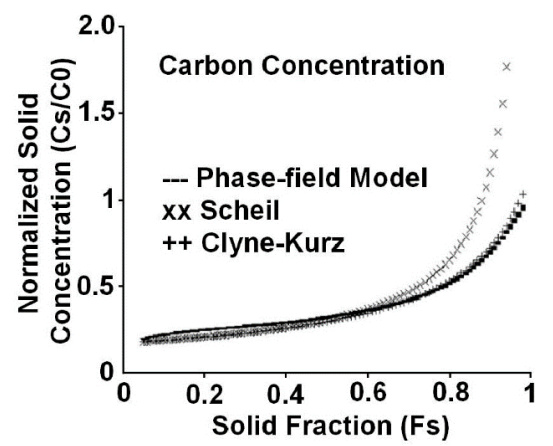

Figure 8. Comparison of carbon concentration as evaluated: via the phase-field model; with Scheil's equation; and with the Clyne-Kurz equation.

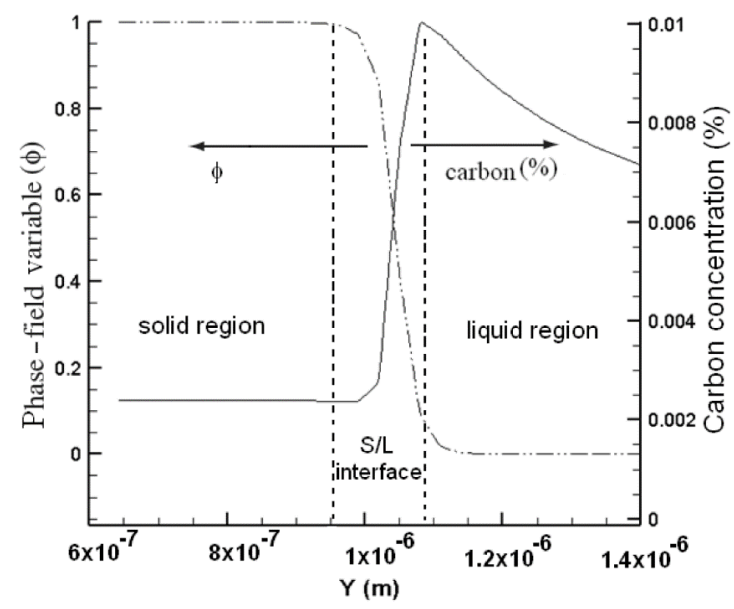

Figure 9. Carbon concentration by region: solid $(\phi=+1)$, liquid $(\phi=0)$, and interface $(0<\phi<+1)$. 


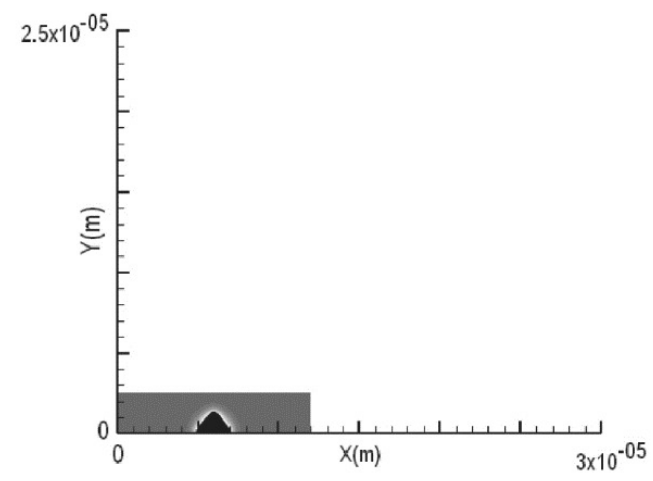

(a)

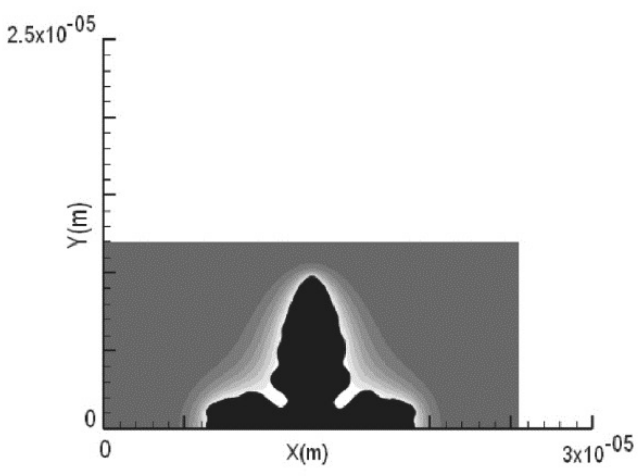

(c)

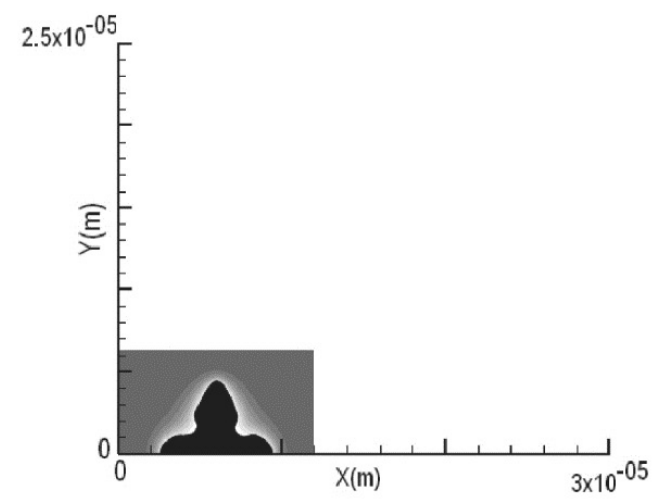

(b)

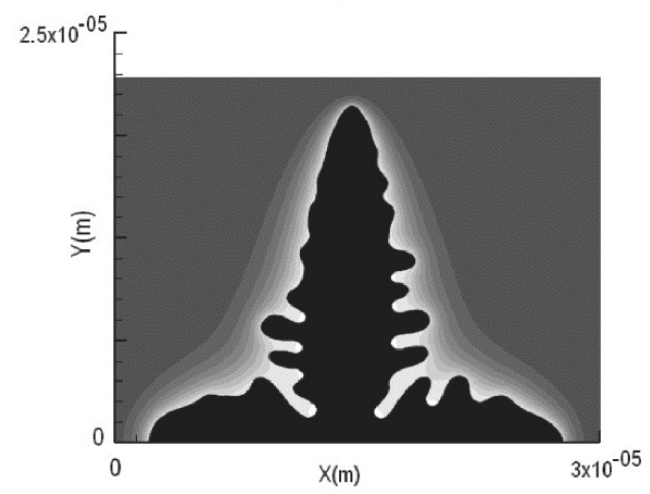

(d)

Figure 10. Development of the adaptive computational domain for dendritic growth for binary alloy (Fe-C). The numerical grids and solidification times are: (a) 85 points, $1.33 \times 10^{-5} \mathrm{sec}$; (b) 205 points, $7.01 \times 10^{-5} \mathrm{sec}$; (c) 400 points, $2.06 \times 10^{-4}$ sec; and (d) 605 points, $1.50 \times 10^{-3} \mathrm{sec}$

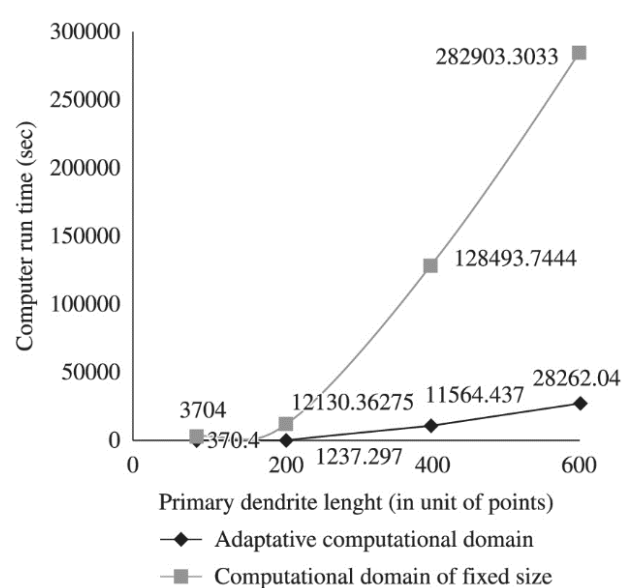

Figure 11. Variation of computer run time as a function of primary dendrite length, in units of points, for binary alloy (Fe-C).

units of domain size) for binary alloy (Fe-C). The run time required to reach a given length for primary dendrite is about tenth of that with the computational domain of fixed size. The efficiency is guaranteed via adaptive computational domain for phase field and concentration calculation due to the small computational domain at the beginning of the calculations.

\section{Conclusions}

In spite of the proven ability of phase-field models for computation of the pattern evolution in solidification, they suffer from low computational efficiency. In the computation of a dendrite with side branches, the computational space should be discretized into a mesh with about two million cells. Such a high number leads to a considerable increase of the run time. In the present study, a phase-field model is introduced with an adaptive computational domain for efficient computational simulation of the dendritic growth in a system for both pure material (Ni) and binary alloy (Fe-C). The method, which is based on the difference in thermal diffusivity for pure material and solute diffusivity in binary alloy, enables us to reduce by about an order of magnitude the run time for simulation of the solidification via the phase-field model.

The phase and thermal fields were calculated adaptively only in the regions that satisfy the condition $\mathbf{T}(\mathbf{I}, \mathbf{J})>\mathbf{T}_{\mathbf{0}}+\mathbf{1 . 1}$, for simulation of solidification in pure material. The phase and concentration fields were calculated in the regions that satisfy the condition $\mathbf{C}(\mathbf{I}, \mathbf{J})>\mathbf{C}_{\mathbf{0}}+\mathbf{1} \times \mathbf{1 0}^{-3}$, for solidification of binary alloy. The computation showed that the dendrite with developed secondary and tertiary arms can be obtained on a personal computer with a much reduced run time. The calculated dendritic morphology displayed a microstructure quite similar to results found in literature and experiments. 


\section{References}

1. Ferreira AF, Silva AJ and Castro JA. Simulation of the solidification of pure nickel via the phase-field method. Materials Research. 2006; 9(4):349-356.

2. Ode M, Kim SG, Kim WT and Suzuki T. Numerical prediction of the secondary dendrite arm spacing using a phase-field model. ISIJ International. 2001; 41(4):345-349. http://dx.doi. org/10.2355/isijinternational.41.345.

3. Ferreira AF and Ferreira LO. Microsegregation in Fe-C-P ternary alloys using a phase-field model. Journal of the Brazilian Society of Mechanical Sciences and Engineering. 2009; 31(3):173-180. http://dx.doi.org/10.1590/S1678-58782009000300002.

4. Salvino IM, Ferreira LO and Ferreira AF. Simulation of microsegregation in multicomponent alloys during solidification. Steel Research International. 2012; 83(8):723-732. http://dx.doi. org/10.1002/srin.201200003.

5. Xu Y, McDonough JM and Tagavi KA. A numerical procedure for solving 2D phase-field model. Journal of Computational Physics. 2006; 218(2):770-793. http://dx.doi.org/10.1016/j. jcp.2006.03.007.
6. Moelans N, Blanpain B and Wollants P. An introduction to phase-field modeling of microstructure evolution. Computer Coupling of Phase Diagrams and Thermochemistry. 2008; 32(2):268-294. http://dx.doi.org/10.1016/j.calphad.2007.11.003.

7. Boettinger WJ, Warren JA, Beckermann C and Karma A. Phasefield simulation of solidification. Annual Review of Materials Research. 2002; 32(1):163-194. http://dx.doi.org/10.1146/ annurev.matsci.32.101901.155803.

8. Kim SG, Kim WT, Lee JS, Ode M and Suzuki T. Large scale simulation of dendritic growth in pure undercooled melt by phase-field model. ISIJ International. 1999; 39(4):335-340. http://dx.doi.org/10.2355/isijinternational.39.335.

9. Stefanescu DM. Science and engineering of casting solidification . 2nd ed. Ohio: Springer; 2009. p. 160.

10. Altundas YB and Caginalp G. Computations of dendrites in 3-D and comparison with microgravity experiments. Journal of Statistical Physics. 2003; 110(3-6):1055-1067. http://dx.doi. org/10.1023/A:1022140725763.

11. Campos MP Fo. and Davies GJ. Solidificação e fundição de metais e suas liga. Rio de Janeiro: Livros Técnicos e Científicos; 1978. p. 59. 